\title{
不同溶剂对甲氨蝶呤/类水滑石复合物性质的影响
}

\author{
张晓晴 曾美桂李淑萍* \\ (江苏省生物功能材料重点实验室 南京师范大学化学与材料科学学院＼cjkstart南京 210097)
}

\begin{abstract}
摘要 采用共沉淀法将甲氨蝶呤(MTX)插层组装到类水滑石(LDHs)层间, 分别考察了不同溶剂(如: 水、乙醇/水、聚 乙二醇- 400 /水、聚乙二醇- 4000 /水)对合成的甲氨蝶呤/类水滑石(MTX/LDHs)纳米复合物性质的影响. 利用透射电镜 (TEM)和原子力显微镜(AFM)观察产物形貌, 利用 X-射线衍射(XRD)、傅里叶变换红外(FTIR)、热重/差式扫描量热 (TG-DSC)和紫外光谱(UV-Vis)等表征手段, 对纳米复合物的结构及热力学性质进行了系统的研究. 在 $\mathrm{pH}=7.4$ 的磷酸 盐缓冲溶液中测定不同时间点药物累积释放量, 考察了不同溶剂中合成的 MTX/LDHs 纳米复合物的药物控释性能. 结 果表明, 短链聚乙二醇的加入保证了纳米粒子的稳定生长, 能有效控制产物形貌, 合成出的产物呈规则的圆片状, 单 分散性好, 药物缓释性能平稳, 释放过程属于药物扩散控制过程. 当聚乙二醇分子链过长时, 由于其自身容易发生缠 绕, 反而不利于纳米粒子的生长.
\end{abstract}

关键词 类水滑石; 聚乙二醇; 形貌规则; 体外释放

\section{Influence of Different Solvents on the Property of Methotrexate/Layered Double Hydroxides}

\author{
Zhang, Xiaoqing Zeng, Meigui Li, Shuping* \\ (Jiangsu Key Laboratory of Biofunctional Materials, College of Chemistry and Materials Science, \\ Nanjing Normal University, Nanjing 210097, China)
}

\begin{abstract}
Methotrexate (MTX) was intercalated into the layered double hydroxides (LDHs) by the coprecipitation method to form MTX/LDHs nanocompounds, the effect of different solvents, i.e. water, mixture of ethanol and water, mixture of polyethylene glycol-400/4000 (PEG-400/4000) and water, on the properties of MTX/LDHs nanocompounds has been examined carefully. The nanocompounds were then characterized by X-ray diffraction (XRD), Fourier transform infrared spectroscopy (FTIR), transmission electron/micrograph (TEM), atomic force microscopy (AFM), thermogravimetry/differential scanning calorimetry (TG-DSC) and UV-visible diffuse spectroscopy (UV-vis). XRD and FTIR investigations demonstrated the successful intercalation of MTX anions as a declining monolayer into the interlayer of LDHs and the interlayer spacing changed accordingly with the variation in the kind of solvents. We thought that the addition of ethanol and PEG just changed the growth environment, especially the property of interlayer water in MTX/LDHs compounds and the hypothesis has been proved by the analysis of TG-DSC. There is no intercalation of PEG molecular into the LDHs interlayers from all the characterization. Compared with the product prepared in other solvents, the particles obtained in the mixture of PEG-400 and water exhibited round plates with the best monodispersity and the most regular morphology. The mechanism how PEG-400 molecules influence the formation of MTX/LDHs nanocompounds is described emphatically: non-ionized PEG-400 molecules will form chain-like structures due to the assembly in water, and the growth of nanocompounds is strictly limited in these structures. Due to the inhibition effect of PEG-400, further agglomeration will be forbidden; as a result the monodispersity will be improved. But when the molecular chain of PEG is too long (i.e. PEG-4000), it goes against the growth of nanocompounds on the contrary. The in vitro release experiment has been carried out in phosphate buffer solution at the $\mathrm{pH}$ value of 7.4, and the result revealed that the release property of MTX/LDHs can be well described by parabolic diffusion equation, or the release mechanism is mainly belongs to drug diffusion. The work reported here will help to establish a general method for the synthesis of drug/LDH nanocompounds with regular morphology and perfect dispersion properties.

Keywords layered double hydroxides; polyethylene glycol; uniform particles; in vitro release
\end{abstract}

\section{1 前言}

水滑石类化合物包括水滑石(Hydrotalcite)和类水滑
石(Hydrotalcite like compound, 简写为 HTlc), 其主体一 般由两种金属的氢氧化物构成, 因此又称为层状双金属 氢氧化物(Layered Double Hydroxides, 简写为 LDHs),

*E-mail: lishuping@njnu.edu.cn; Tel: 0086-025-83598280.

Received November 15, 2012; published December 6, 2012.

Supporting information for this article is available free of charge via the internet at http//sioc-journal.cn

Project supported by the National Natural Science Foundation of China (No. 21073093), Specialized Research Fund for the Doctoral Program of Higher Education of China (No. 20103207120006) and the Priority Academic Program Development of the Jiangsu Higher Education Institutions of China.

项目受国家自然科学基金(No. 21073093)、高等学校博士点专项科研基金(No. 20103207120006)和江苏高校优势学科建设工程项目资助. 
其化学通式可表示为: $\left[\mathrm{M}_{1-x}^{2+} \mathrm{M}_{x}^{3+}(\mathrm{OH})_{2}\right]\left[\mathrm{A}^{n-}\right]_{x / n} \bullet m \mathrm{H}_{2} \mathrm{O}$, $\mathrm{M}^{2+}$ 表示 +2 价金属离子, $\mathrm{M}^{3+}$ 表示 +3 价金属离子, $\mathrm{A}$ 表 示层间阴离子 ${ }^{[1]}$. 其中层间阴离子具有可交换性, 因此 可将各种阴离子(如: 有机、无机、配合物、同多和杂多 阴离子等)通过交换而插层组装到 LDHs 层间, 从而得到 具有不同性能的复合材料 ${ }^{[2]}$. LDHs 具有安全性、稳定 性、生物相容性和良好的生物可降解性, 且有利于细胞 吸收及药物释放, 近年来已受到生物医学材料界的特别 关注, 是一类极具应用前景的新型纳米药物载体 ${ }^{[3]}$. 把 客体药物组装到 LDHs 层间可得到药物/LDHs 纳米复合 物, 药物/LDHs 纳米复合物的形成一方面可降低药物的 毒副作用、提高其溶解度、稳定性、增强其靶向性等; 另 一方面, 药物与 LDHs 层板间存在静电作用、氢键作用 或范德华力以及空间位阻效应等 ${ }^{[4]}$, 可与周围环境中阴 离子进行缓慢离子交换, 进而实现药物的有效控释. Ambrogi 等 ${ }^{[5}$ 利用离子交换法成功地将消炎药布洛芬组 装到镁铝类水滑石层间, 研究结果表明 LDHs 作为药物 载体可以达到非常好的缓释效果. Tyner 等 ${ }^{[6]}$ 利用阴离子 表面活性剂修饰喜树碱分子表面, 再将其与 LDHs 进行 阴离子交换, 成功地将非离子型、水溶性差的抗癌药物 一喜树碱组装到 LDHs 层间, 极大提高了喜树碱的疗效.

甲氨蝶呤(Methotrexatum, 简称 MTX) 是一种抗代 谢类抗肿瘤药, 对许多恶性肿瘤均具有良好的治疗作用, 但 MTX 的毒副作用较大, 主要表现为对胃肠道、血液、 肝脏、肾脏的毒性 ${ }^{[7]}$. MTX 分子顶端含有两个羧基(MTX 分子式见图 1), 可以很容易地组装到 LDHs 层间, 从而 对其起到保护和缓释作用, 达到提高药物疗效、降低其 毒副作用的目的. Choy 等 ${ }^{[8]}$ 已成功实现 MTX 组装到 LDHs 层间, 证明其在少剂量时仍具有很高的药效, 其 药效比纯 MTX 高出 5000 多倍. 在药物/LDHs 纳米复合 物制备过程中, 由于复合物尺寸小, 表面能高, 极易发 生团聚现象; LDHs 表面羟基的存在也使颗粒之间容易 因氢键作用发生团聚, 从而直接影响到其应用. 因此, 改善 MTX/LDHs 纳米复合物的单分散性是满足其临床 应用的前提之一。

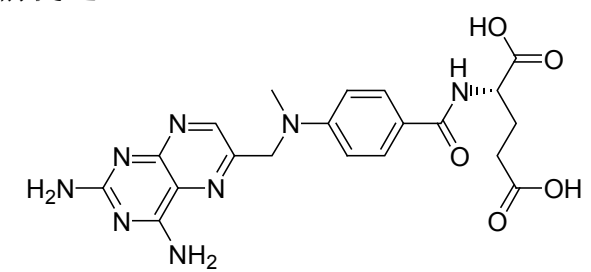

图 1 MTX 分子结构图

Figure 1 Structural formula of the MTX molecule

聚乙二醇(Polyethylene Glycol, 简称 PEG)具有非常 好的生物相容性, 对人体无害, 是一种无毒且具有良好 生物相容性的溶剂, 在医药、食品等行业都有着广泛的 应用 ${ }^{[9]}$. 由于链长的影响, 不同分子量的聚乙二醇往往 有不同的物理性质及不同的应用. PEG-200 600 的水 溶液是良好的溶剂, 可提高难溶性药物的溶解度, 并且
对在水中不稳定的药物有稳定作用, 故可作为注射用溶 剂. 其中 PEG-400 呈液状, 与各种溶剂均具有广泛的相 容性, 是很好的溶剂和增溶剂, 被广泛用于制备各种液 体制剂, 如口服液、滴眼液等. PEG-4000, PEG-6000 在 医药、化妆品等工业生产中通常被用作基质，起调节粘 度、熔点的作用。

本文在前期工作 ${ }^{[10]}$ 的基础上, 系统研究了不同溶 剂对 MTX/LDHs 纳米复合物性质的影响, 首次选用不 同分子链长度的 PEG-400 与 PEG-4000 作为溶剂, 以期 得到形貌规则和单分散性良好的 MTX/LDHs 纳米复合 物, 并与其他溶剂下得到的 MTX/LDHs 纳米复合物的 性能进行了比较. 尤为重要的是, 对不同溶剂下合成产 物的机理和缓释机理进行了初步的探索性研究.

\section{2 结果与讨论}

\section{$2.1 \mathrm{XRD}$ 分析}

图 2 为不同溶剂中合成的 MTX/LDHs 纳米复合物 的 XRD 图, 从图中可以看出, 合成的产物均具有 $\mathrm{LDHs}$ 特有的(003), (006), (009)和(110)晶面特征衍射峰, 且峰 型尖锐, 对称性良好, 说明所合成产物均为晶型规整的 LDHs 化合物 ${ }^{[11]}$. LDHs 为层状结构, 属于六方晶系, $d_{003}$ 可用来表示层间距. 与母体 $\mathrm{NO}_{3}-\mathrm{LDHs}$ 相比 ${ }^{[12]}$, 纳米复 合物的 $d_{003}$ 衍射峰均向低角度方向移动 $\left(2 \theta: \mathrm{a}-4.26^{\circ}\right.$, b-3.68 ${ }^{\circ}, \mathrm{c}-3.56^{\circ}, \mathrm{d}-2.97^{\circ}$ ), 这表明 MTX 阴离子已成功插 层到 LDHs 层间, 导致了 LDHs 层间距的增大. 我们对 PEG-400, PEG-4000 与 Mg-Al-NO ${ }_{3}$-LDHs 的反应历程也 进行了进一步的分析, XRD 结果表明, PEG-400 和 PEG-4000 不能插层到 LDHs 层间, 详见 Supporting information. 值得注意的是，在 PEG-4000 中得到的复合 物峰强度最低, 这表明 PEG-4000 的加入降低了 LDHs 层状结构的有序排列, 不利于复合物晶型的成长和发 育.

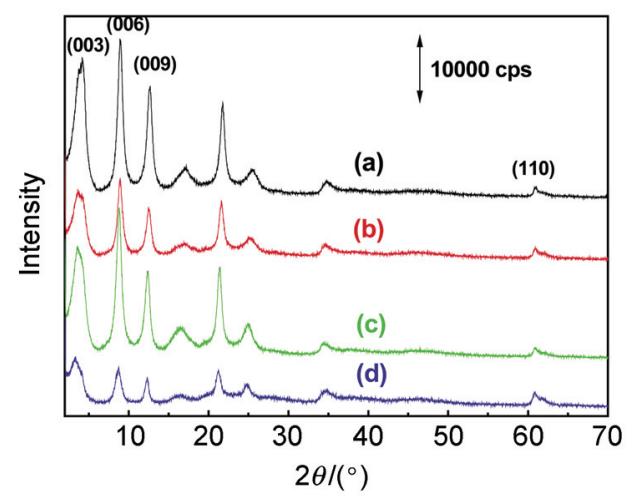

图 2 不同溶剂中合成的 MTX/LDHs 纳米复合物 XRD 图

Figure 2 XRD patterns of MTX/LDHs nanocompounds in different solvents

(a) water; (b) ethanol/water; (c) PEG-400/water; (d) PEG-4000/water

根据 Bragg 方程 $2 d \sin \theta=n \lambda$ (其中 $n$ 为衍射级数, $\theta$ 
为衍射角, $d$ 为层间距), 可求出所有复合物的层间距, 见表 1. 由于 $\mathrm{LDHs}$ 层板厚度为 $0.48 \mathrm{~nm}^{[13]}$, MTX 分子长 度为 $2.12 \mathrm{~nm}$, 由此可求出纳米复合物的层间通道以及 计算出 MTX 分子在 LDHs 层板间的倾斜角度，见表 1 . 据此可模拟出 MTX 分子在 LDHs 层板间排列方式, 见 图 3. 从图 3 可以看出 MTX 在 $\mathrm{LDHs}$ 层间是以单层倾斜 方式排列的，从表 1 可以看出当含有羟基的溶剂加入后, 得到产物的层间距比水作溶剂时的要大. 影响 LDHs 层 间距数值的因素有: 层间阴离子体积、电荷密度及层间 水的位置和含量等 ${ }^{[14]}$, 而造成 $\mathrm{MTX} / \mathrm{LDHs}$ 纳米复合物 层间距不同的原因应与层间水的性质不同有关. PEG 分 子或乙醇分子附着在纳米粒子表面后, 形成了一层溶剂 化层, 当其从 $\mathrm{LDHs}$ 颗粒周围分离时, 该溶剂化层被破 坏, 使得渗透压降低, 从而产生一种相互作用的有效吸 引力 ${ }^{[15]}$, 使层间水分子与 LDHs 层板上的羟基之间的相 互作用逐渐增强, 这种强作用力使层间水在类水滑石层 中的含量增加, 从而造成了产物层间距的增加. 因此, 随着溶剂中美基数目的增加, 产物的层间距逐渐增大, 其中 PEG-4000/水作溶剂时合成的产物层间距最大, 这 说明在该溶剂下合成复合物时，层板上的羟基与层间水 分子之间的相互作用力最强.

表 1 不同溶剂中合成的 MTX/LDHs 纳米复合物的晶体结构数据

Table 1 Crystal structure data of MTX/LDHs nanocompounds in different solvents

\begin{tabular}{cccccc}
\hline Solvent & $\begin{array}{c}\text { Mean } \\
\text { particle } \\
\text { size/nm }\end{array}$ & $\begin{array}{c}\text { Basal } \\
\text { spacing/nm }\end{array}$ & $\begin{array}{c}\text { Interlayer } \\
\text { spacing } / n m\end{array}$ & $\begin{array}{c}\text { Tilting } \\
\left.\text { angle } /{ }^{(}\right)\end{array}$ & $\begin{array}{c}\text { Drug-loading } \\
\text { capacity/\% }\end{array}$ \\
\hline $\mathrm{H}_{2} \mathrm{O}$ & 274 & 2.08 & 1.60 & 50 & 50.88 \\
$\mathrm{EtOH} / \mathrm{H}_{2} \mathrm{O}$ & 254 & 2.42 & 1.94 & 66 & 51.49 \\
$\mathrm{PEG}-400 / \mathrm{H}_{2} \mathrm{O}$ & 231 & 2.48 & 2.00 & 70 & 51.85 \\
PEG-4000/ $\mathrm{H}_{2} \mathrm{O}$ & 203 & 2.66 & 2.18 & 90 & 56.84 \\
\hline
\end{tabular}

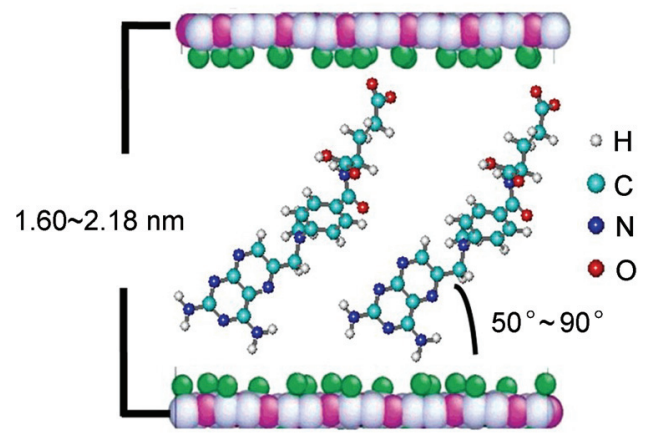

图 3 不同溶剂中合成的 MTX/LDHs 纳米复合物中 MTX 在 LDHs 层 间排列方式模拟图

Figure 3 Scheme model of the arrangement of MTX in MTX/LDHs nanocompounds in different solvents

\subsection{FTIR 分析}

图 4 是不同溶剂中合成的 MTX/LDHs 纳米复合物 的红外光谱图, 从中可以看出, 所有复合物红外峰的峰 型与峰位非常相似. 为了比较, 母体 $\mathrm{NO}_{3}-\mathrm{LDHs}$ 和 MTX
的红外光谱也示于图 4 中. 从中可以看出, $\mathrm{NO}_{3}-\mathrm{LDHs}$ 的 红外谱图与复合物的有明显的不同: $3460 \mathrm{~cm}^{-1}$ 处共同 存在的比较宽的特征峰是物理吸附水或结晶水的 $\mathrm{OH}^{-}$ 伸缩振动谱带, $\mathrm{NO}_{3}-\mathrm{LDHs}$ 在 $1386 \mathrm{~cm}^{-1}$ 处有一个强烈的 吸收峰, 这是 $\mathrm{NO}_{3}^{-}$的不对称伸缩峰, 而 MTX/LDHs 纳 米复合物中没有此峰出现, 表明 $\mathrm{LDHs}$ 层板内的 $\mathrm{NO}_{3}^{-}$已 被 MTX 阴离子所取代 ${ }^{[16]}$. MTX/LDHs 纳米复合物位于 $1624 \mathrm{~cm}^{-1}$ 处较强的峰为 MTX 中的 $-\mathrm{COOH}$ 的吸收峰, $1450 \mathrm{~cm}^{-1}$ 处的峰为 $\mathrm{MTX}$ 芳香环中 $\mathrm{C}=\mathrm{C}$ 的伸缩振动峰, $1208 \mathrm{~cm}^{-1}$ 和 $1100 \mathrm{~cm}^{-1}$ 处的峰则对应于 MTX 芳香环中 伯胺和叔胺的 $\mathrm{C}-\mathrm{N}$ 吸收峰.

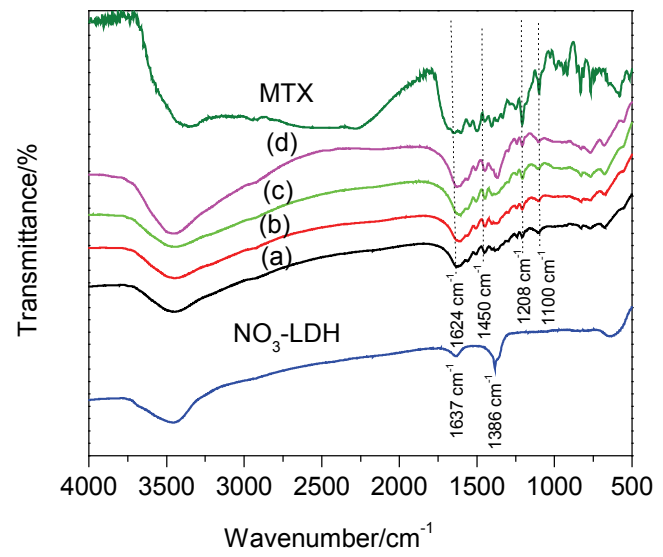

图 4 不同溶剂中合成的 MTX/LDHs 纳米复合物的 FTIR 图 Figure 4 FTIR spectra of MTX/LDHs nanocompounds in different solvents

(a) water; (b) ethanol/water; (c) PEG-400/water; (d) PEG-4000/water

\subsection{TEM 分析}

不同溶剂下得到的 MTX/LDHs 纳米复合物的 TEM 图和粒径分布见图 5, 其中粒径分布图是由测量多张 TEM 图中粒子 ( >100 个)粒径得到的统计结果. 由图可 知, 所有的 MTX/LDHs 纳米复合物皆呈圆片状. 在水中 合成的纳米复合物, 粒子生长不均匀、粒径分布范围较 广; 在乙醇/水中合成的 $\mathrm{MTX} / \mathrm{LDHs}$ 纳米复合物, 其单 分散性和粒子形貌都得到了一定程度的改善, 晶体结构 趋于完整，但粒径分布范围仍较宽. PEG 作为常用的聚 合物类溶剂，其加入在一定程度上增加了溶液的粘度， 从而提高了纳米体系的均匀分散性和稳定性. 其中, 在 PEG-400/水中合成的 MTX/LDHs 纳米复合物的形貌最 规则, 呈圆形, 晶体粒子生长最完整, 单分散性得到了 明显的改善; 而以 PEG-4000/水为溶剂则出现了粒径分 布宽化的现象. 这是因为 PEG-4000 的分子链较长, 容 易造成自身的相互缠绕，从而失去了保护纳米粒子的功 效 ${ }^{[17]}$. 线性 PEG 的分子式为 $\mathrm{H}-\left(\mathrm{O}-\mathrm{CH}_{2}-\mathrm{CH}_{2}\right)_{n}-\mathrm{OH}$, 由于 PEG-400 分子链长度适中, 在溶液中可组装成链状结构, 可以将 $\mathrm{MTX} / \mathrm{LDHs}$ 纳米复合物严格地限制在由 PEG-400 分子组装成的空间内生长, 起到空间位阻效 应 $^{[18]}$. 在反应过程中, PEG-400 既是分散剂又是稳定剂, 
同时起到了控制颗粒尺寸和表面修饰的双重作用，通过 配位或电荷作用包覆在粒子表面以控制粒子成核及生 长, 从而能够有效地改善 MTX/LDHs 纳米复合物的形 貌和单分散性 ${ }^{[19,20]}$.

纳米粒子在溶液中的生长机制主要是基于奥氏熟 化(Ostwald ripening)和取向连接过程(Oriented attach- ment ${ }^{[21]}$. 奥氏熟化理论是基于纳米粒子表面化学势高, 溶解性增加，因而导致较小粒子溶解，大粒子优先生长, 该过程主要受扩散、界面的溶解与沉淀的影响. 取向连 接过程则是将纳米粒子自身作为结构单元，以与其晶体 学相匹配的方式进行聚集生长, 以消除自由表面, 释放 出表面能的过程. PEG-400 覆盖在纳米粒子表面会使得
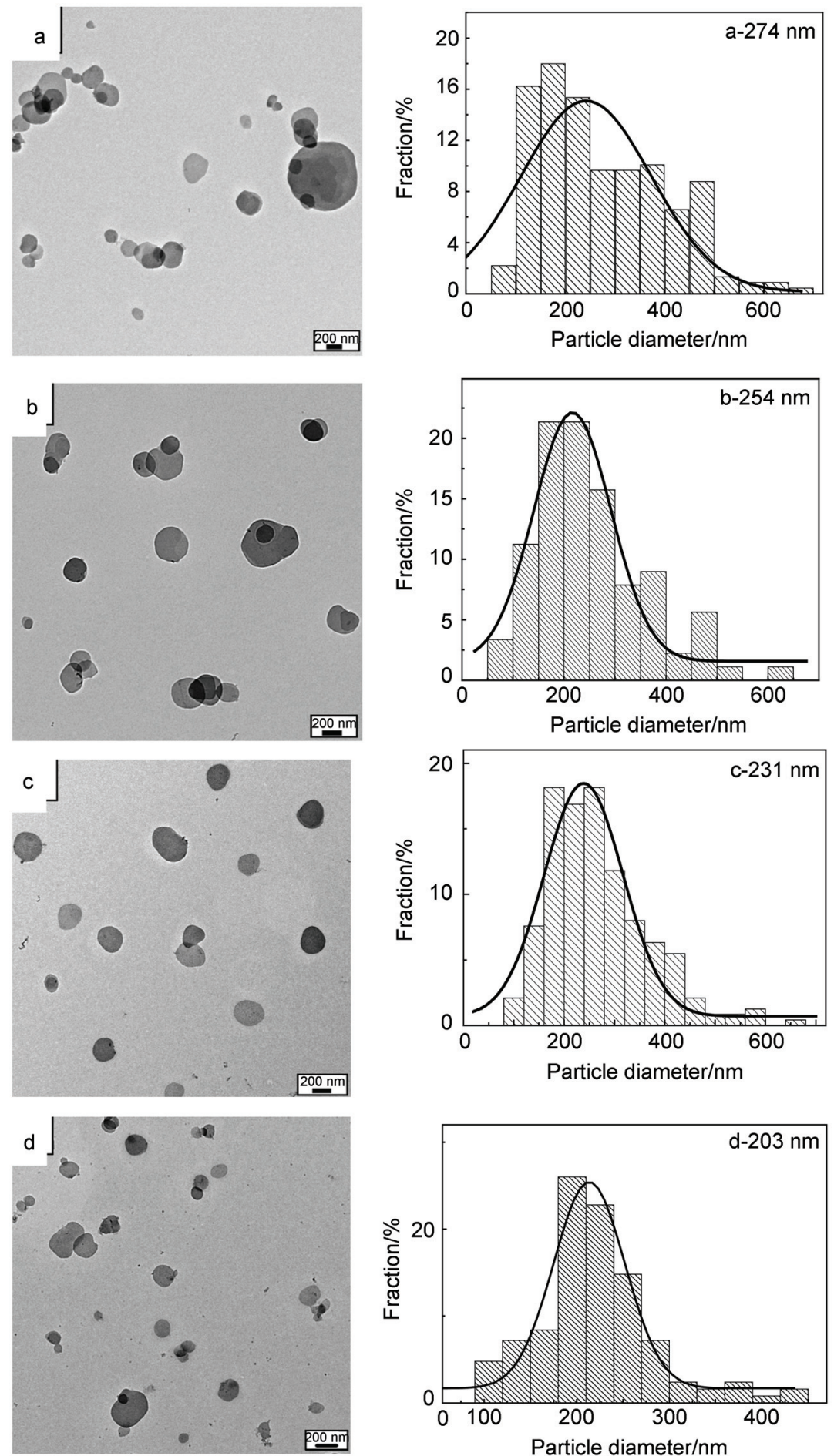

图 5 不同溶剂中合成的 MTX/LDHs 纳米复合物 TEM 图及粒径分布图

Figure 5 TEM images and particle size distributions of MTX/LDHs nanocompounds in different solvents (a) water; (b) ethanol/water; (c) PEG-400/water; (d) PEG-4000/water 
粒子生长初期的奥氏熟化过程受到抑制, 而使取向生长 占优势, 保证了纳米粒子成核过程的进行, 从而对粒子 生长起到一定的稳定作用; 后期的水热处理过程, 则使 得奥氏熟化与取向生长同时进行, 提高了纳米粒子生长 的稳定性，从而在一定程度上保证了粒子粒径的均一 性 ${ }^{[21]}$.

由复合物的平均粒径数据可知(表 1), 乙醇和 PEG 的加入会使产物粒径减小. 这是由于乙醇和 PEG 的加 入都会起到一定的隔离作用, 阻碍了纳米粒子之间的直 接接触与结合. 其中, PEG-400 具有链式分子结构, 能 够包裹在纳米粒子的表面, 在其表面形成一层亲水大分 子保护膜, 当溶液中加入 PEG-400 后, 表面羟基的大量 存在使得粒子之间发生空间排斥作用而使得粒子相互 远离, 可以有效阻止颗粒的团聚, 并使其缓慢生长. 而 PEG-4000 由于分子链较长, 在水溶液中容易发生分子 链间的相互缠绕, 从而失去了保护纳米粒子的功效, 见 图 6.

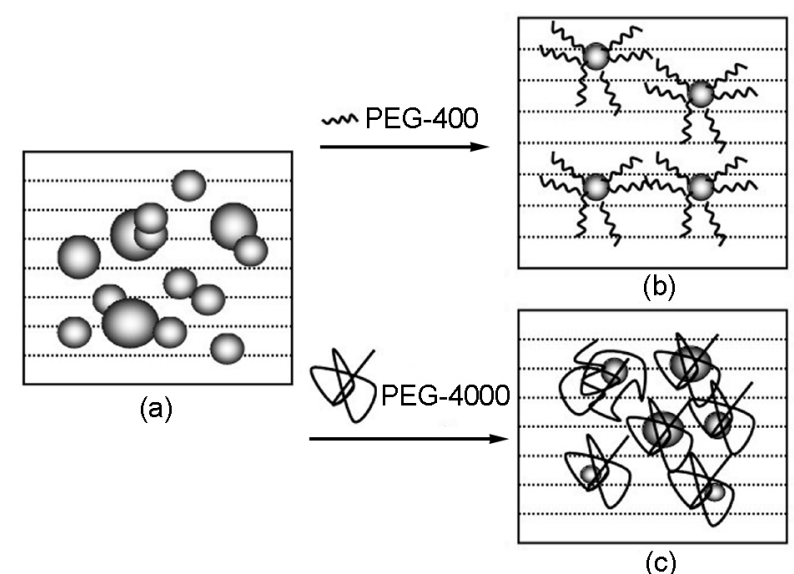

图 6 加入 PEG 前后 MTX/LDHs 纳米复合物合成过程示意图 Figure 6 Scheme of the MTX/LDHs nanocompounds in different conditions

(a) without PEG; (b) with PEG-400; (c) with PEG-4000

\subsection{AFM 分析}

通过 AFM 图像可以观察到纳米粒子的形貌及厚度, $\mathrm{MTX} / \mathrm{LDHs}$ 纳米复合物的 $\mathrm{AFM}$ 图见图 7. 从 AFM 图中 可以看出, 产物粒子皆呈现圆片状, 水作溶剂时形成的 粒子出现明显的团聚, 乙醇/水和 PEG-4000/水中合成的 粒子单分散性差, 而 PEG-400/水中合成的粒子单分散 性良好, 粒径均匀. 图中所标出粒子的厚度分别为: 水 作溶剂-7 nm、乙醇/水为溶剂-3 nm、 PEG-400/水为溶 剂-15 nm、PEG-4000/水为溶剂-30 nm, 由于水、乙醇/ 水、PEG-4000/水作溶剂合成的产物单分散性差，其厚度 测量结果只是一个大概值. PEG-400/水作溶剂合成产物 粒径均一, 粒子厚度均匀, 从 AFM 图中得到其平均粒 径为 $240 \mathrm{~nm}$, 与 $\mathrm{TEM}$ 图结果基本一致. LDHs 晶格属于 斜方六面体对称，因此一方面基于晶体生长的取向性， LDHs 层板会优先沿 $a-b$ 轴方向生长而形成六角形 ${ }^{[22]}$;
另一方面通过静电相互作用的层片堆积 ( $c$ 轴)生长属于 动力学有利而热力学上不利的过程 ${ }^{[23]}$, 这两个过程相 互作用使得产物最终形成了六角片状结构. 从 AFM 结 果可知, 以 PEG 为溶剂时得到的粒子厚度有所增加, 这 说明 PEG 的加入在保证了晶体稳定性的基础上促进了 晶粒在各个方向充分生长.

\subsection{TG-DSC 分析}

不同溶剂中合成的 $\mathrm{MTX} / \mathrm{LDHs}$ 纳米复合物的 TG-DSC 曲线如图 8 所示. 一般来说, 药物/LDHs 纳米 复合物的失重过程可分为两个步骤: (1) 室温到 $230{ }^{\circ} \mathrm{C}$ 左右, 为表面吸附水和插层水分子的脱离过程; (2) $230 \sim 705{ }^{\circ} \mathrm{C}$ 为层间着基和层间药物离子的脱去过程 ${ }^{[24]}$. 从图 8 的 $\mathrm{TG}$ 曲线中(为方便看清其他样品失重变化, 将 其中母体 $\mathrm{NO}_{3}-\mathrm{LDHs}$ 的 $\mathrm{TG}$ 曲线上移)可以看出, 水、乙 醇/水、PEG-400/水、PEG-4000/水中合成的 MTX/LDHs 纳米复合物的总失重分别为 $49.19 \%, 50.10 \%, 51.54 \%$, $55.86 \%$, 远远高于 $\mathrm{NO}_{3}-\mathrm{LDHs}$ 的总失重值(约 $\left.41 \%\right)^{[25]}$, 这说明 MTX 已成功插层到 LDHs 层间. 在 DSC 图中(为 看清 DSC 的峰变化, 分别对曲线进行了等间隔移动), 四组样品在 $200{ }^{\circ} \mathrm{C}$ 附近出现了吸热峰, 对应于表面水 和层间插层水分子脱离所产生的吸热峰, 其位置分别位 于 $196{ }^{\circ} \mathrm{C}$ (水为溶剂), $212{ }^{\circ} \mathrm{C}$ (乙醇/水为溶剂), $227{ }^{\circ} \mathrm{C}$ (PEG-400/水为溶剂)和 $230{ }^{\circ} \mathrm{C}(\mathrm{PEG}-4000 /$ 水为溶剂). 一 般说来吸热峰的峰值位置与分子间的作用力密切相关: 分子间的作用力强, 脱离过程中所需的热量相应地就会 增加, 导致吸热峰向高温移动 ${ }^{[26]}$. 因此, PEG 的加入使 层间水分子与 LDHs 层板上的羟基之间的相互作用增强, 而且 PEG 的链长越长, 这种作用力就越强, 而水为溶剂 会导致层间水分子与 $\mathrm{LDHs}$ 层板间的作用力最弱，这与 我们前面的分析是一致的. 同样地, $360{ }^{\circ} \mathrm{C}$ 附近出现了 明显的吸热峰, 对应于层间药物分子脱去所产生的吸热 峰, 其位置基本相同(分别为 $359{ }^{\circ} \mathrm{C}, 359{ }^{\circ} \mathrm{C}, 360{ }^{\circ} \mathrm{C}$, $\left.361{ }^{\circ} \mathrm{C}\right)$, 表明不同溶剂下组装于层间的药物分子与层 板间的相互作用力基本相同，即 PEG 或乙醇的加入只 影响到复合物层间水分子与 LDHs 层板间的相互作用力, 对药物分子与 LDHs 层板之间的作用力影响不大. 对比 纯 MTX 在 185 204 ${ }^{\circ} \mathrm{C}$ 即发生分解 ${ }^{[27]}$, MTX/LDHs 纳米 复合物中的 MTX 的分解温度在 $360{ }^{\circ} \mathrm{C}$ 左右, 这也充分 表明 LDHs 对插层化合物的热稳定性具有显著的增强作 用, 可以作为优良的药物载体和储存器.

\section{6 缓释性能分析}

从表 1 可以看出, 不同溶剂中制得的 MTX/LDHs 纳米复合物的载药量分别为 $50.88 \%, 51.49 \%, 51.85 \%$, $56.84 \%$; 结合层间距的数值, 我们可以得到如下结论: 复合物的载药量随着层间距的增加而增大, 这与 $\mathrm{Li}$ 等 ${ }^{[28]}$ 的研究结果是一致的. MTX/LDHs 纳米复合物的缓释曲 线见图 9, 从中可以看出 4 种复合物都具有非常明显的 

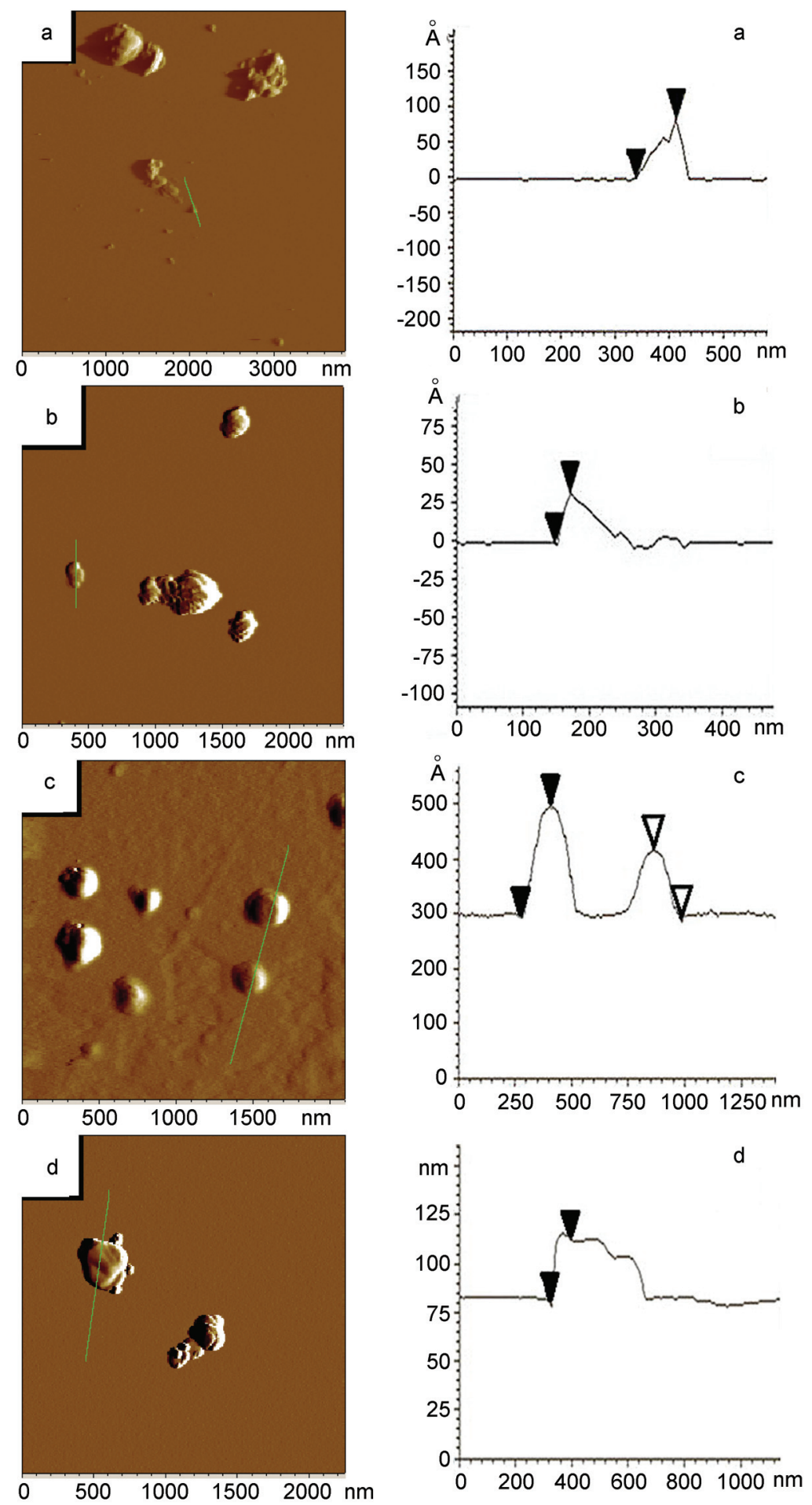

图 7 不同溶剂中合成的 $\mathrm{MTX} / \mathrm{LDHs}$ 纳米复合物 $\mathrm{AFM}$ 图及粒子厚度

Figure 7 AFM images and thickness of MTX/LDHs nanocompounds in different solvents

(a) water; (b) ethanol/water; (c) PEG-400/water; (d) PEG-4000/water

缓释效果, 缓释曲线平稳, 无药物的突释现象发生. 这 一结论对我们后期药物临床试验中的给药量等方面提 供了一定的理论依据.

\section{7 缓释动力学拟合}

药物控释体系释放机理的研究对于药物控释载体
的设计与药物研制起到十分重要的作用. 通常药物的释 放动力学可以通过研究一定温度下, 药物释放率 $\left(M_{t} / M_{\infty}\right)$ 与时间 $(t)$ 的关系来确定. 对于 MTX/LDHs 纳米复合物, 通常选用以下动力学方程:

$$
\ln \left(1-M_{t} / M_{\infty}\right)=-k_{1} t \quad \text { 一级方程 }
$$




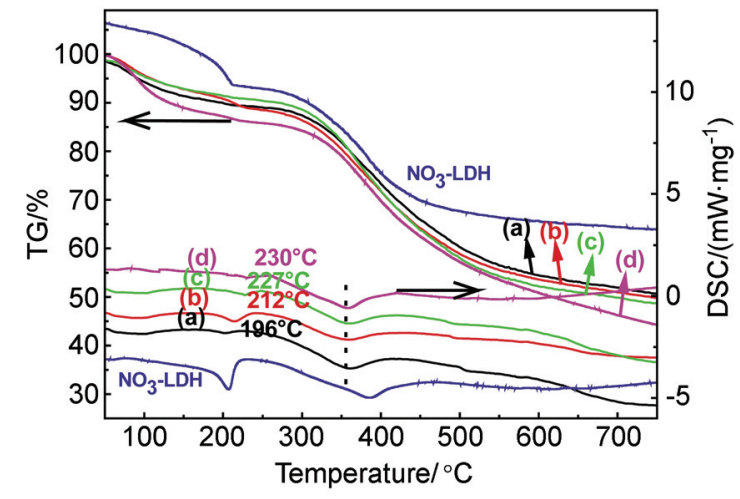

图 8 不同溶剂中合成的 MTX/LDHs 纳米复合物 TG-DSC 图 Figure 8 The TG-DSC curves for MTX/LDHs nanocompounds in different solvents

(a) water; (b) ethanol/water; (c) PEG-400/water; (d) PEG-4000/water; (e) $\mathrm{NO}_{3}-\mathrm{LDH}$

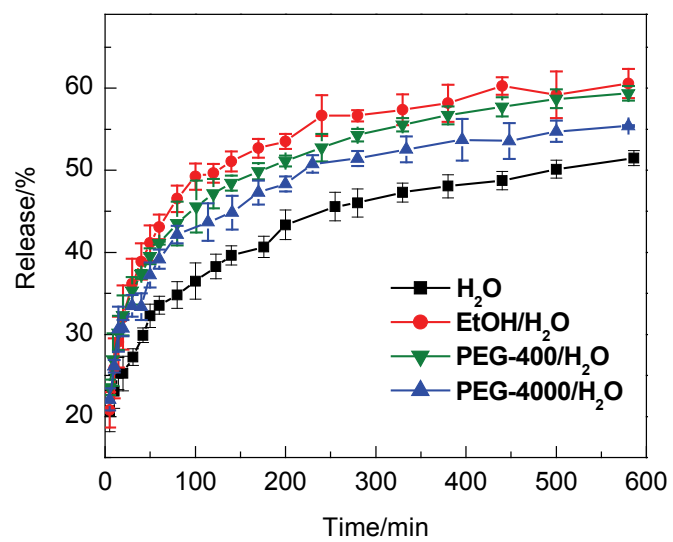

图 9 不同溶剂中合成的 $\mathrm{MTX} / \mathrm{LDHs}$ 纳米复合物缓释曲线

Figure 9 Release profiles of MTX/LDHs nanocompounds in different solvents

$$
\begin{array}{lr}
M_{t} / M_{\infty}=k_{\mathrm{H}} t^{1 / 2} & \text { Higuchi 方程 } \\
M_{t} / M_{\infty}=k t^{n} & \text { Ritger-Peppas 方程 } \\
\left(M_{t} / M_{\infty}\right) / t=k_{\mathrm{P}} t^{-0.5}+b & \text { Parabolic 扩散方程 }
\end{array}
$$

式中, $t$ 为时间, $k$ 为动力学方程的速率常数, $M_{t}$ 和 $M_{\infty}$ 分 别为时间为 $t$ 时和平衡时的释放率. 采用上述公式对药 物释放数据进行动力学拟合, 当拟合因子 $R$ 大于 0.95 时, 可认为释放行为较为符合此方程描述的机理.

分别采用以上 4 组动力学方程对实验所得的释放数 据进行拟合, 图 10 为不同复合物的缓释动力学拟合曲 线, 表 2 为动力学拟合数据. 根据 MTX/LDHs 纳米复合 物释放曲线的特征，我们采用了分段拟合: 快速释放期: 0 200 min; 缓慢释放期: $200 \sim 600 \mathrm{~min}$, 拟合数据见表 2. 结果表明在 $\mathrm{pH}=7.4$ 的磷酸盐缓冲溶液中, 4 种不同 溶剂中合成的 MTX/LDHs 纳米复合物释放过程都符合 Parabolic 扩散方程, 此方程多用于描述粘土类物质的表 面扩散和体相扩散行为 ${ }^{[29]}$, 这说明 MTX 从 LDHs 层间 释放的过程属于扩散控制过程. 从拟合数据可以看出: MTX/LDHs 纳米复合物作为缓释药物具有较明显的缓
释控释的效果; 其中以 PEG-400/水作为溶剂时得到的 复合物的线性最好, 能很好地满足药物载体的控药-缓 释的目的.

\section{3 结论}

通过比较不同溶剂中合成 MTX/LDHs 纳米复合物 的性能可以得到如下结论：由 PEG-400/水作溶剂合成 出的 $\mathrm{MTX} / \mathrm{LDHs}$ 纳米复合物具有较高的结晶度, 药物 插层后复合物中无杂质残留, 产物形貌为规则的圆片状, 单分散性良好, 且溶剂加入不会对药物分子与 LDHs 层 板的相互作用力产生影响. 缓释实验结果表明, 4 种纳 米复合物皆具有非常明显的缓释效果, 缓释曲线都非常 平稳, 无药物的突释现象发生, 其释药机理符合 Parabolic 扩散方程, 释药过程为药物扩散控制过程. 本 文分析了不同溶剂对粒子的形貌与结构的影响机制, 为 合成单分散性良好的类水滑石纳米复合物提供了很好 的理论依据.

\section{4 实验部分}

\subsection{MTX/LDHs 纳米复合物制备}

将 $0.032 \mathrm{~mol} \mathrm{Mg}\left(\mathrm{NO}_{3}\right)_{2} \bullet 6 \mathrm{H}_{2} \mathrm{O}$ 和 $0.016 \mathrm{~mol} \mathrm{Al}\left(\mathrm{NO}_{3}\right)_{3} \cdot$ $9 \mathrm{H}_{2} \mathrm{O}$, 溶于 $20 \mathrm{~mL}$ 溶剂(a: 水, b: 乙醇/水, c: PEG-400/ 水, d: PEG-4000/水, 其中乙醇、PEG 与水体积比皆为 $1: 3)$ 中配成混合盐溶液 A, 将 $0.008 \mathrm{~mol}$ MTX 溶于 15 $\mathrm{mL} 、 10 \%$ 的氨水混合液中配成溶液 B. 将溶液 B 加入到 三口烧瓶中, 通氮气 $1 \sim 2 \mathrm{~min}$, 同时打开磁力搅拌器, 将溶液 $\mathrm{A}$ 以 $0.05 \mathrm{~mL} / \mathrm{s}$ 的滴速滴加到溶液 $\mathrm{B}$ 中, 最终以 $10 \%$ 的氨水调节 $\mathrm{pH}$ 至 9.5 , 保温 $60{ }^{\circ} \mathrm{C}$ 下反应 $1 \mathrm{~h}$, 最后 用去离子水和乙醇交替离心洗涤三次(离心速度 10000 $\mathrm{r} / \mathrm{min}$, 离心时间 $1 \mathrm{~min}$ ). 合成的产物放到 $50 \mathrm{~mL}$ 的高压 反应釜(内祄为聚四氟乙烯)中, 经 $100{ }^{\circ} \mathrm{C}, 48 \mathrm{~h}$ 的水热 处理 ${ }^{[30]}$ 后即制备出了 MTX/LDHs 纳米复合物.

\section{2 载药量测量与缓释性能研究}

精密称取 $0.01 \mathrm{~g}$ MTX, 分别溶于 $\mathrm{pH}=1.2$ 的盐酸和 $\mathrm{pH}=7.4$ 的磷酸盐缓冲溶液中, 在 $100 \mathrm{~mL}$ 容量瓶中定容, 摇匀得到 $100 \mu \mathrm{g} / \mathrm{mL}$ 对照品. 精密吸取对照品溶液 1.0 $\mathrm{mL}, 2.5 \mathrm{~mL}, 4.0 \mathrm{~mL}, 5.5 \mathrm{~mL}, 7.0 \mathrm{~mL}, 8.5 \mathrm{~mL}, 10.0 \mathrm{~mL}$ 分 别置于 $50 \mathrm{~mL}$ 的容量瓶中, 用上述溶液稀释至刻度摇匀, 在 $306 \mathrm{~nm}$ 处测定吸光度 ${ }^{[31]}$. 以质量浓度 $C$ 为横坐标, 吸 光度 $A$ 为纵坐标, 分别做出标准工作曲线和线性方程.

(1) $\mathrm{pH}=1.2$ 的盐酸溶液

$$
A=0.04208 C+0.00245(R=0.9999, n=7)
$$

(2) $\mathrm{pH}=7.4$ 的磷酸盐缓冲溶液

$$
A=0.05185 C+0.00062(R=0.99999, n=7)
$$

精密称取 $0.01 \mathrm{~g} \mathrm{MTX/LDHs}$ 纳米复合物, 溶于 $\mathrm{pH}$ $=1.2$ 的盐酸中, 在 $500 \mathrm{~mL}$ 容量瓶中定容并摇匀, 得到 

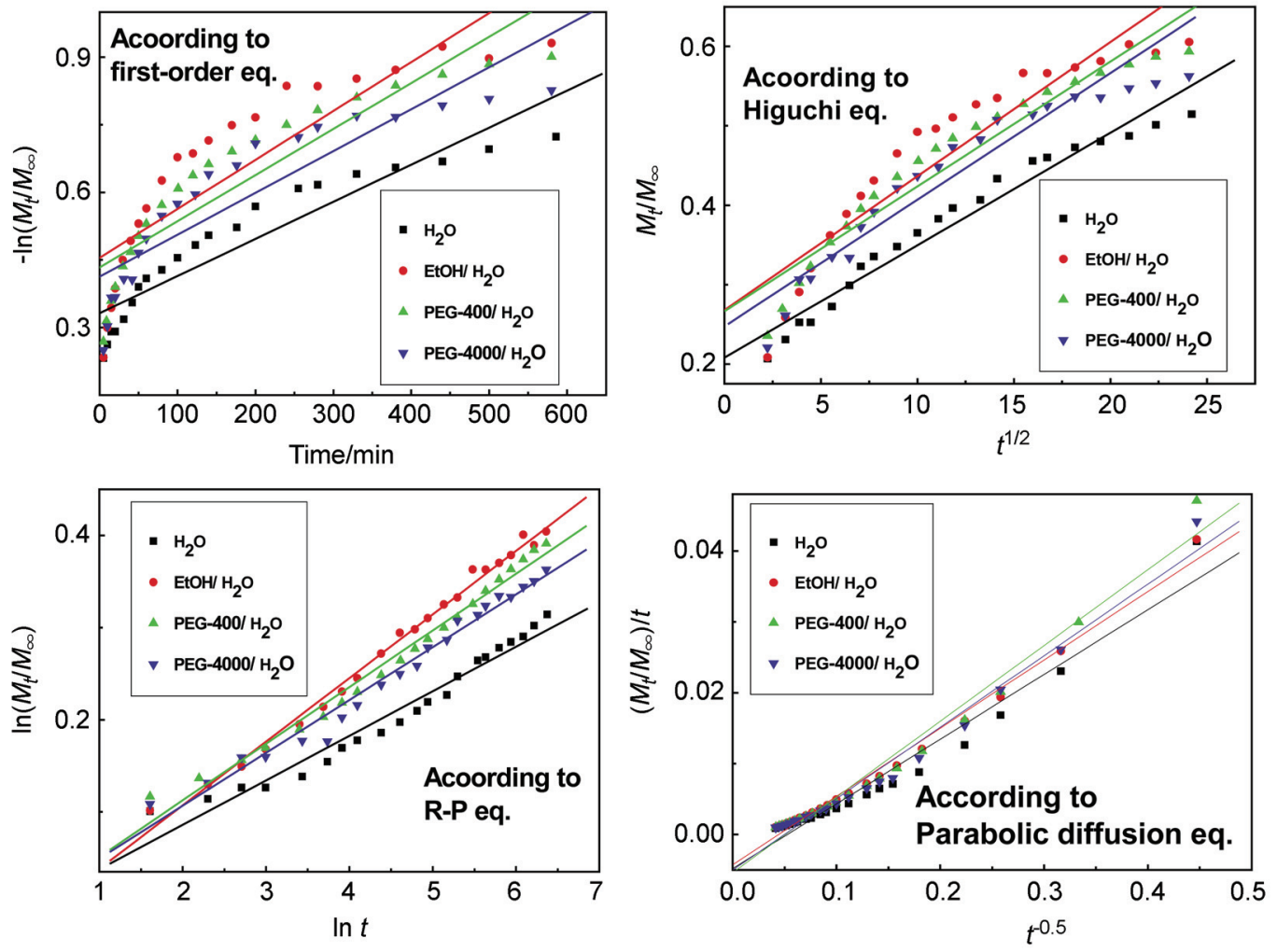

图 10 不同溶剂中合成的 $\mathrm{MTX} / \mathrm{LDHs}$ 纳米复合物缓释动力学曲线

Figure 10 Plots of different kinetic models for the release of MTX from the MTX/LDHs nanocompounds

表 2 不同溶剂中合成的 MTX/LDHs 纳米复合物的缓释动力学拟合数据

Table 2 The release data of MTX/LDHs nanocompounds fitting to different kinetic equations

\begin{tabular}{|c|c|c|c|c|c|c|}
\hline \multirow{3}{*}{ Kinetic models } & \multicolumn{6}{|c|}{ Samples } \\
\hline & \multicolumn{3}{|c|}{$\mathrm{H}_{2} \mathrm{O}$} & \multicolumn{3}{|c|}{$\mathrm{EtOH} / \mathrm{H}_{2} \mathrm{O}$} \\
\hline & $\mathrm{I}^{a}$ & $\mathrm{II}^{b}$ & $\mathrm{~W}^{c}$ & $\mathrm{I}^{a}$ & $\mathrm{II}^{b}$ & $\mathrm{~W}^{c}$ \\
\hline First-order eq. & 0.9659 & 0.9968 & 0.9340 & 0.9286 & 0.9289 & 0.8730 \\
\hline Higuchi eq. & 0.9919 & 0.9965 & 0.9795 & 0.9693 & 0.9336 & 0.9318 \\
\hline Ritger-Peppas eq. & 0.9785 & 0.9915 & 0.9797 & 0.9796 & 0.9920 & 0.9920 \\
\hline Parabolic diffusion eq. & 0.9923 & 0.9986 & 0.9799 & 0.9918 & 0.9975 & 0.9944 \\
\hline \multirow{3}{*}{ Kinetic models } & \multicolumn{6}{|c|}{ Samples } \\
\hline & \multicolumn{3}{|c|}{ PEG-400/ $\mathrm{H}_{2} \mathrm{O}$} & \multicolumn{3}{|c|}{$\mathrm{PEG}-4000 / \mathrm{H}_{2} \mathrm{O}$} \\
\hline & $\mathrm{I}^{a}$ & $\mathrm{II}^{b}$ & $\mathrm{~W}^{c}$ & $\mathrm{I}^{a}$ & $\mathrm{II}^{b}$ & $\mathrm{~W}^{c}$ \\
\hline First-order eq. & 0.9464 & 0.9815 & 0.9145 & 0.9590 & 0.9750 & 0.8959 \\
\hline Higuchi eq. & 0.9821 & 0.9887 & 0.9625 & 0.9847 & 0.9577 & 0.9795 \\
\hline Ritger-Peppas eq. & 0.9944 & 0.9976 & 0.9877 & 0.9749 & 0.9814 & 0.9882 \\
\hline Parabolic diffusion eq. & 0.9965 & 0.9995 & 0.9960 & 0.9853 & 0.9991 & 0.9892 \\
\hline
\end{tabular}

${ }^{a}$ For release in $0 \sim 200$ min. ${ }^{b}$ For release in $200 \sim 600 \min ^{c}{ }^{c}$ For the whole release process $(0 \sim 600 \mathrm{~min})$.

浓度为 $20 \mu \mathrm{g} / \mathrm{mL}$ 的溶液, 在 $306 \mathrm{~nm}$ 处测定吸光度值, 并根据线性方程(1)计算此时 MTX 浓度, 求出载药量.

根据载药量计算出缓释实验所需的 MTX/LDHs 纳 米复合物的量, 称量后溶解于 $\mathrm{pH}=7.4$ 的磷酸盐缓冲溶 液中, 定容至 $500 \mathrm{~mL}$ (按照中国药典 2005 年版第二部附 录 XIVE 方法配置), 在 $(37 \pm 0.5){ }^{\circ} \mathrm{C}$ 下恒温摚拌, 在预 设定的时间间隔内, 取 $3 \mathrm{~mL}$ 溶液高速离心分离, 取上
清液在 $306 \mathrm{~nm}$ 处测其吸光度，按线性方程(2)计算出相 应药物浓度及释放百分比，绘制 MTX 的累积释放曲线.

\section{References}

[1] Rives, V. Layered Double Hydroxides: Present and Future, Nova Science Publishers, New York, 2001, pp. 229 250.

[2] Feng, Y. J.; Williams, G. R.; Leroux, F.; Taviot-Gueho, C.; O'Hare, D. Chem. Mater. 2006, 18, 4312.

[3] Kwak, S. Y.; Jeong, Y. J.; Park, J. S.; Choy, J. H. Solid State Ionics 
2002, 151, 229.

[4] Kwak, S. Y.; Kriven, W. M.; Wallig, M. A.; Choy, J. H. Biomaterials 2004, 25, 5995.

[5] Ambrogi, V.; Fardella, G.; Grandolini, G.; Nocchetti, M.; Perioli, L. Int. J. Pharm. 2001, 220, 23.

[6] Tyner, K. M.; Schiffman, S. R.; Giannelis, E. P. J. Controlled Release 2004, 95, 501.

[7] Zheng, H. Medicinal Chemistry, 6th ed., People's Medical Publishing House, Beijing, 2007, pp. 241 242. (郑虎, 药物化学, 第 6 版, 人民卫生出版社, 北京, 2007, pp. $241 \sim 242$.)

[8] Oh, J. M.; Park, M.; Kim, S. T.; Jung, J. Y.; Kang, Y. G.; Choy, J. H. J. Phys. Chem. Solids 2006, 67, 1024.

[9] Lu, J.; Liu, Q.-F.; Luo, G.-A.; Wang, Y.-M. Chin. J. Org. Chem. 2009, 29, 1167. (路娟, 刘清飞, 罗国安, 王义明, 有机化学, 2009, 29, 1167.)

[10] Wang, J.-Q.; Li, X.; Li, S.-P.; Zhong, H. Acta Chim. Sinica 2011, 69, 137. (王继芹, 李金鍑, 李淑萍, 仲慧, 化学学报, 2011, 69, 137.)

[11] Jin, L.; Liu, Q.; Sun, Z.-Y.; Ni, X.-Y.; Wei, M. Ind. Eng. Chem. Res. 2010, 49, 11176.

[12] Aisawa, S.; Ohnuma, Y.; Hirose, K.; Takahashi, S.; Hirahara, H.; Narita, E. Appl. Clay Sci. 2005, 28, 137.

[13] Aisawa, S.; Takahashi, S.; Ogasawara, W. J. Solid State Chem. 2001, 162, 52

[14] Cavani, F.; Trifiro, F.; Vaccari, A. Catal. Today 1991, 11, 173.

[15] Zhu, W.-X.; Sun, D.-J.; Liu, S.-Y.; Wang, N.; Zhang, J.; Luan, L.-Y. Colloids Surf. A: Physicochem. Eng. Aspects 2007, 301, 106.

[16] Oh, J. M.; Choi, S. J.; Kim, S. T.; Choy, J. H. Bioconjugate Chem. 2006, 17, 1411.

[17] Yao, G.-Y.; Li, Y.-B.; Lu, B.-D.; Wei, Z.-X. Chin. J. Rare Met. 2007, 31, 192. (姚根有, 李延斌, 逯宝娣, 卫芝贤, 稀有金属, 2007, 31, 192.)
[18] Hu, D.-W.; Wang, Y.-M. J. Chin. Ceram. Soc. 2008, 36, 1488. (胡大 为, 王燕民, 硅酸盐学报, 2008, 36, 1488.)

[19] Wen, Y.-M.; Lu, Z.-Q. Chem. Res. Appl. 2002, 14, 563. (温燕梅, 卢 泽勤, 化学研究与应用, 2002, 14, 563.)

[20] Wang, J.; Peng, Z.-M.; Huang, Y.-J.; Chen, Q.-W. J. Cryst. Growth 2004, 263, 616.

[21] Gilbert, B.; Zhang, H.; Huang, F.; Finnegan, M. P.; Waychunas, G. A.; Banfield, J. F. Geochem. Trans. 2003, 4, 20.

[22] Liu, Z.-P.; Ma, R.-Z.; Osada, M.; Iyi, N.; Ebina, Y.; Takada, K.; Sasaki, T. J. Am. Chem. Soc. 2006, 128, 4872.

[23] Xu, Z.-P.; Stevenson, G. S.; Lu, C. Q.; Lu, G.-Q. J. Phys. Chem. B 2006, 110, 16923 .

[24] Yang, Q.-Z.; Sun, D.-J.; Zhang, C.-G.; Wang, X.-J.; Zhao, W.-A. Langmuir 2003, 19, 5570.

[25] Valente, J. S.; Sánchez-Cantú, M.; Lima, E.; Figueras, F. Chem. Mater. 2009, 21, 5809.

[26] Li, C.; Wang, L.-Y.; Evans, D. G.; Duan, X. Ind. Eng. Chem. Res. 2009, 48, 2162.

[27] Choy, J. H.; Jung, J. S.; Oh, J. M.; Park, M.; Jeong, J.; Kang, Y. K.; Han, O. J. Biomaterials 2004, 25, 3059.

[28] Li, Y.-H.; Xu, J.; Zhang, S.-J.; Li, D.-X.; Zheng, B.; Hou, W.-G. Chin. J. Inorg. Chem. 2009, 25, 2124. (李艳红, 徐洁, 张少杰, 李 东祥, 郑斌, 侯万国, 无机化学学报, 2009, 25, 2124.)

[29] Kong, X.-G.; Jin, L.; Wei, M.; Duan, X. Appl. Clay Sci. 2010, 49, 324.

[30] Zhang, X.-Q.; Qi, F.-L.; Li, S.-P.; Wei, S.-H.; Zhou, J.-H. Appl. Surf. Sci. 2012, 259, 245.

[31] Wang, Y.-S.; Han, Y.-L.; Li, Y.-X.; Wang, Y.-M.; Li, R.-S. Chem. J. Chin. Univ. 2007, 28, 1092. (王银松, 韩月莲, 李英霞, 王玉玫, 李荣珊, 高等学校化学学报, 2007, 28, 1092.) 